\title{
Crystal structure of $\left(2,2^{\prime}\right.$-bipyridine- $\left.\kappa^{2} N, N^{\prime}\right)$ bis(4- (dimethylamino)phenyldiphenylphosphane-K $P$ ) copper(I) tetrafluoroborate, $\mathrm{C}_{50} \mathrm{H}_{48} \mathrm{BCuF}_{4} \mathrm{~N}_{4} \mathrm{P}_{2}$
}
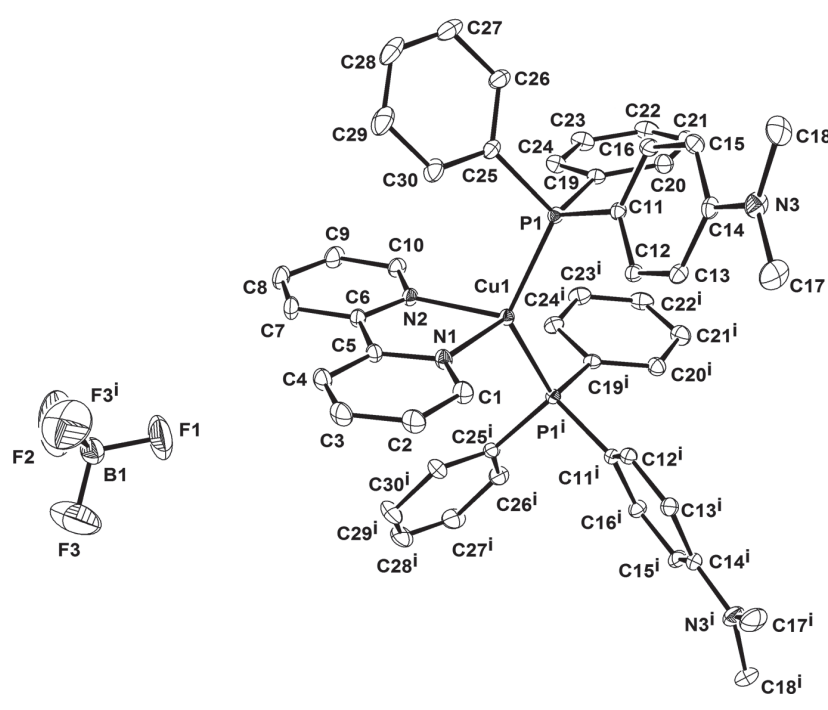

https://doi.org/10.1515/ncrs-2018-0289

Received August 6, 2018; accepted October 9, 2018; available online December 11, 2018

\begin{abstract}
$\mathrm{C}_{50} \mathrm{H}_{48} \mathrm{BCuF}_{4} \mathrm{~N}_{4} \mathrm{P}_{2}$, orthorhombic, Pnma (no. 62), $a=24.166(2) \AA, \quad b=19.5652(18) \AA, \quad c=9.6477(9) \AA, \quad Z=4$, $V=4561.6(7) \AA^{3}, \quad R_{\mathrm{gt}}(F)=0.0494, \quad w R_{\mathrm{ref}}\left(F^{2}\right)=0.1546$, $T=298 \mathrm{~K}$.
\end{abstract}

CCDC no.: 1042163

The crystal structure is shown in the figure. Tables 1 and 2 contain details on crystal structure and measurement conditions and a list of the atoms including atomic coordinates and displacement parameters.

\footnotetext{
*Corresponding author: Mohammed Fettouhi, Department of Chemistry, King Fahd University of Petroleum and Minerals, P.O. Box 5048, Dhahran 31261, Saudi Arabia,

e-mail: fettouhi@kfupm.edu.sa

Atif Fazal: Centre for Refining and Petrochemicals, Research Institute, King Fahd University of Petroleum and Minerals, Dhahran, 31261, Saudi Arabia
}

๑ Open Access. ( 2019 Atif Fazal et al., published by De Gruyter. (cc) BY License.
Table 1: Data collection and handling.

\begin{tabular}{ll}
\hline Crystal: & Plate, orange \\
Size: & $0.54 \times 0.40 \times 0.16 \mathrm{~mm}$ \\
Wavelength: & Mo $K \alpha$ radiation $(0.71073 \AA$ A $)$ \\
$\mu:$ & $0.61 \mathrm{~mm}^{-1}$ \\
Diffractometer, scan mode: & Bruker SMART APEX, $\omega$-scans \\
$\theta_{\max }$, completeness: & $50^{\circ},>99 \%$ \\
$N(h k l)_{\text {measured }}, N(h k l)_{\text {unique }}, R_{\text {int }}:$ & $45154,5832,0.036$ \\
Criterion for $I_{\text {obs }}, N(h k l)_{\mathrm{gt}}:$ & $I_{\text {obs }}>2 \sigma\left(I_{\text {obs }}\right), 4398$ \\
$N(\text { param })_{\text {refined }}:$ & 306 \\
Programs: & Bruker programs [1], SHELX [2, 3], \\
& ORTEP-3 [4] \\
\hline
\end{tabular}

\section{Source of material}

The reaction of stoichiometric amounts, with the ratio $(1 / 1 / 2)$ respectively of 2,2'-bipyridine, $\left[\mathrm{Cu}\left(\mathrm{CH}_{3} \mathrm{CN}\right)_{4}\right] \mathrm{BF}_{4}$ and 4-(dimethylamino)phenyldiphenylphosphine, in dichloromethane for $12 \mathrm{~h}$ followed by evaporation of the solvent yields a yellow powder. Re-crystallization from dimethylformamide affords crystals of the title compound.

\section{Experimental details}

All hydrogen atoms were placed in calculated positions using a riding model with $\mathrm{C}-\mathrm{H}$ distances of $0.93 \AA$, or $0.96 \AA$. The isotropic displacement parameters were $U_{\text {iso }}(\mathrm{H})=1.5 U_{\text {eq }}(\mathrm{C})$ for the methyl atoms and $U_{\text {iso }}(\mathrm{H})=1.2 U_{\text {eq }}(\mathrm{C})$ for others.

\section{Discussion}

Heteroleptic copper(I) complexes based on diimine and phosphine ligands are of current interest owing to their chemical, photophysical and catalytic properties [5]. The reported structures of copper(I) complexes based on 4(dimethylamino)phenyldiphenylphosphine are scarce in literature [6] and the structures of the corresponding heteroleptic complexes with $2,2^{\prime}$-bipyridine have not been reported to date.

The copper(I) ion is bonded to the two nitrogen atoms of 2,2'-bipyridine and the phosphorus atoms of two 4(dimethylamino)phenyldiphenylphosphine molecules. The complex, including the copper(I) ion and the $2,2^{\prime}$-bipyridine ligand are located on a mirror plane. The geometry is distorted 
Table 2: Fractional atomic coordinates and isotropic or equivalent isotropic displacement parameters $\left(\AA^{2}\right)$.

\begin{tabular}{|c|c|c|c|c|}
\hline Atom & $x$ & $y$ & $z$ & $U_{\text {iso }} * / U_{\text {eq }}$ \\
\hline Cu1 & $0.40157(2)$ & 0.2500 & $0.26915(4)$ & $0.03952(14)$ \\
\hline N1 & $0.32491(12)$ & 0.2500 & $0.3719(3)$ & $0.0519(7)$ \\
\hline N2 & $0.42698(13)$ & 0.2500 & $0.4782(3)$ & $0.0479(7)$ \\
\hline N3 & $0.25946(13)$ & $0.02825(15)$ & $-0.2266(3)$ & $0.0747(8)$ \\
\hline P1 & $0.42314(2)$ & $0.14640(3)$ & $0.17719(6)$ & $0.04038(16)$ \\
\hline $\mathrm{C} 1$ & $0.27461(16)$ & 0.2500 & $0.3161(5)$ & $0.0634(10)$ \\
\hline $\mathrm{H} 1$ & 0.2717 & 0.2500 & 0.2200 & $0.076^{*}$ \\
\hline $\mathrm{C} 2$ & $0.22640(17)$ & 0.2500 & $0.3937(5)$ & $0.0722(12)$ \\
\hline $\mathrm{H} 2$ & 0.1920 & 0.2500 & 0.3507 & $0.087^{\star}$ \\
\hline C3 & $0.23060(19)$ & 0.2500 & $0.5343(6)$ & $0.0758(13)$ \\
\hline H3 & 0.1989 & 0.2500 & 0.5890 & $0.091^{*}$ \\
\hline $\mathrm{C} 4$ & $0.28144(19)$ & 0.2500 & $0.5943(5)$ & $.0681(11)$ \\
\hline $\mathrm{H} 4$ & 0.2847 & 0.2500 & 0.6903 & $0.082^{\star}$ \\
\hline C5 & $0.32844(16)$ & 0.2500 & $0.5123(4)$ & $0.0508(8)$ \\
\hline $\mathrm{C} 6$ & $0.38523(17)$ & 0.2500 & $0.5694(4)$ & $0.0522(8)$ \\
\hline $\mathrm{C} 7$ & $0.3957(2)$ & 00 & $3(4)$ & $0.0719(13)$ \\
\hline $\mathrm{H} 7$ & 0.3663 & 00 & 747 & $0.086^{*}$ \\
\hline $\mathrm{C} 8$ & $0.4487(3)$ & 00 & 1(5) & $0.0829(15)$ \\
\hline $\mathrm{H} 8$ & 0.4558 & 0.2500 & 0.8548 & $0.099 *$ \\
\hline C9 & $0.4917(2)$ & & $0.6664(5)$ & $753(13)$ \\
\hline H9 & & & & 0.090 * \\
\hline $\mathrm{C} 10$ & $0.47881(17)$ & 00 & $0.5262(4)$ & $0.0592(9)$ \\
\hline $\mathrm{H} 10$ & 0.5077 & 0.2500 & 0.4625 & 0.071 * \\
\hline C11 & $0.37850(10)$ & 0.112 & (2) & $0.0424(5)$ \\
\hline $\mathrm{C} 12$ & 10) & 0.1486 & $.0017(3)$ & $0.0468(5)$ \\
\hline $\mathrm{H} 12$ & 269 & 25 & 362 & $0.056^{*}$ \\
\hline C13 & $0.29335(11)$ & 226 & $-0.0881(3)$ & $.0520(6)$ \\
\hline $\mathrm{H} 13$ & 532 & 93 & -0 & $0.062^{*}$ \\
\hline C14 & $0.29873(11)$ & 0.056 & -0.14 & $20(6)$ \\
\hline C15 & 12) & $0.01979(14)$ & -0.10 & $0.0585(7)$ \\
\hline $\mathrm{H} 15$ & 0.3520 & -0.0236 & -0 . & $0.070^{*}$ \\
\hline C16 & $8461(12)$ & $.04667(13)$ & $-0.0122(3)$ & $0.0529(6)$ \\
\hline H16 & 0.4152 & 0.0206 & 0.0126 & $0.064^{*}$ \\
\hline C17 & $1112(18)$ & $.0668(2)$ & $-0.2656(5)$ & 991(14) \\
\hline H17A & 902 & 81 & 842 & $0.149^{*}$ \\
\hline H17B & 0.1887 & 0.0399 & -0 & $0.149 *$ \\
\hline $\mathrm{H} 17 \mathrm{C}$ & 0.2223 & & -0 . & $0.149^{*}$ \\
\hline $\mathrm{C} 18$ & 6051(19) & -0.043 & -0.2 & $896(12)$ \\
\hline $18 \mathrm{~A}$ & 0.2919 & -0 & -0 & $0.134^{*}$ \\
\hline $18 \mathrm{~B}$ & 0.2272 & -0 & -0 & $0.134^{*}$ \\
\hline $\mathrm{H} 18 \mathrm{C}$ & 0.2632 & -0.0698 & -0.1751 & $0.134^{*}$ \\
\hline C19 & $358(10)$ & 14208(11) & $0.1106(3)$ & $.0466(5)$ \\
\hline $\mathrm{C} 20$ & $692(12)$ & 3145 & $6(3)$ & $0.0608(7)$ \\
\hline $\mathrm{H} 20$ & 0.4791 & 23 & -0.0904 & $0.073^{*}$ \\
\hline $\mathrm{C} 21$ & $167(14)$ & $.13448(17)$ & $-0.0704(4)$ & $.0760(9)$ \\
\hline $\mathrm{H} 21$ & 0.5704 & 0.1279 & -0.1633 & 0.091 * \\
\hline $\mathrm{C} 22$ & 027 & 472 & & $0.0773(10)$ \\
\hline $\mathrm{H} 22$ & 0.6 & 93 & -0 & $0.093^{*}$ \\
\hline $\mathrm{C} 23$ & $0.59065(13)$ & $0.15701(17)$ & $0.1609(4)$ & $0.0724(9)$ \\
\hline $\mathrm{H} 23$ & 0.6189 & 0.1652 & 0.2242 & $0.087^{*}$ \\
\hline $\mathrm{C} 24$ & $3650(12)$ & $5470(14)$ & $2048(3)$ & $0.0600(7)$ \\
\hline $\mathrm{H} 24$ & 0.5283 & 0.1616 & & $0.072^{*}$ \\
\hline $\mathrm{C} 25$ & $0.42184(12)$ & $0.07785(13)$ & $0.3068(3)$ & $0.0520(6)$ \\
\hline $\mathrm{C} 26$ & $0.45628(15)$ & $0.02112(15)$ & $0.3015(3)$ & $0.0687(8)$ \\
\hline $\mathrm{H} 26$ & 0.4837 & 0.0180 & 0.2343 & $0.082^{*}$ \\
\hline $\mathrm{C} 27$ & $0.44915(19)$ & $0.03175(18)$ & $0.3994(4)$ & $0.0910(12)$ \\
\hline $\mathrm{H} 27$ & 0.4718 & -0.0701 & 0.3962 & $0.109^{*}$ \\
\hline $\mathrm{C} 28$ & $0.4093(2)$ & $-0.0268(2)$ & $0.4984(5)$ & $0.1041(15)$ \\
\hline
\end{tabular}

Table 2 (continued)

\begin{tabular}{lrrrr}
\hline Atom & $\boldsymbol{x}$ & $\boldsymbol{y}$ & $\boldsymbol{z}$ & $\boldsymbol{U}_{\text {iso }}{ }^{*} \boldsymbol{U}_{\text {eq }}$ \\
\hline H28 & 0.4047 & -0.0621 & 0.5619 & $0.125^{*}$ \\
C29 & $0.3764(2)$ & $0.0288(2)$ & $0.5054(4)$ & $0.1064(14)$ \\
H29 & 0.3499 & 0.0322 & 0.5750 & $0.128^{*}$ \\
C30 & $0.38211(16)$ & $0.08114(18)$ & $0.4085(3)$ & $0.0752(9)$ \\
H30 & 0.3587 & 0.1188 & 0.4128 & $0.090^{*}$ \\
B1 & $0.1546(3)$ & 0.2500 & $0.9675(7)$ & $0.0918(19)$ \\
F1 & $0.1796(3)$ & 0.2500 & $0.8544(5)$ & $0.237(4)$ \\
F2 & $0.1900(3)$ & 0.2500 & $1.0590(7)$ & $0.359(8)$ \\
F3 & $0.1238(3)$ & $0.2992(3)$ & $0.9858(7)$ & $0.283(3)$ \\
\hline
\end{tabular}

tetrahedral with two $\mathrm{N}-\mathrm{Cu}-\mathrm{P}$ bond angles of $107.85(3)^{\circ}$ and $112.73(3)^{\circ}$ and a $2,2^{\prime}$-bipyridine bite angle of $78.78(12)^{\circ}$. The $\mathrm{P}-$ $\mathrm{Cu}-\mathrm{P}$ bond angle is $126.17(3)^{\circ}$. The $\mathrm{Cu}-\mathrm{N}$ and $\mathrm{Cu}-\mathrm{P}$ bond distances are similar to those reported for other copper(I) complexes [7]. Both intra and intermolecular non-classical hydrogen bonding interactions take place. The 2,2'-bipyridine ligand is engaged in $\mathrm{CH}_{\text {phenyl }} \cdots \mathrm{N}_{\text {bipy }}(\mathrm{C} \cdots \mathrm{N}$ : 3.599(4) $\AA$ ) interactions with two phosphine phenyl groups in addition to $\mathrm{CH}_{\text {bipy }}$. F F (C...F: 3.325(7) $\AA$ ) interactions with the counterion $\mathrm{BF}_{4}{ }^{-}$. The nitrogen atom of the dimethylamino group is essentially coplanar with the three carbon atoms, the deviation from the mean plane being 0.059(3) A. The N3-C14 bond distance (1.375(3) $\AA$ ) is significantly shorter than N3-C17 and N3-C18 bond distances (1.440(5) Å and 1.446(5) $\AA$ ).

Acknowledgements: The authors gratefully acknowledge the support of CRP and King Fahd University of Petroleum \& Minerals (KFUPM), Dhahran, Saudi Arabia.

\section{References}

1. Bruker. APEX3, SAINT-Plus, XPREP. Bruker AXS Inc., Madison, WI, USA (2016)

2. Sheldrick, G. M.: SHELXT - Integrated space-group and crystal-structure determination. Acta Crystallogr. A71 (2015) 3-8.

3. Sheldrick, G. M.: Crystal structure refinement with SHELXL. Acta Crystallogr. C71 (2015) 3-8.

4. Farrugia, L. J.: ORTEP-3 for windows - a version of ORTEP-III with a graphical user interface (GUI). J. Appl. Cryst. 30 (1997) 565-565.

5. Sandroni, M.; Pellegrin, Y.; Odobel, F.: Heteroleptic bis-diimine copper $(I)$ complexes for applications in solar energy conversion Martina. C. R. Chimie. 19 (2016) 79-93.

6. Shan, X. C.; Jiang, F. L.; Chen, L.; Wu, M. Y.; Pan, J.; Wan, X. Y.; Hong, M. C.: Using cuprophilicity as a multi-responsive chromophore switching color in response to temperature, mechanical force and solvent vapors. J. Mater. Chem. C, 1 (2013) 4339-4349.

7. Navarro, M.; Corona, O. A.; Gonzalez, T.; Capparelli, M. V.: (2,2'Bipyridine)bis(triphenylphosphine)copper(I) nitrate chloroform solvate hemihydrate. Acta Crystallogr. E64 (2008) m533-m534. 